\title{
AN APPLICATION OF LYAPUNOV'S DIRECT METHOD TO THE STUDY OF OSCILLATIONS OF A DELAY DIFFERENTIAL EQUATION OF EVEN ORDER
}

\author{
RAYMOND D. TERRY
}

(Received 9 March 1976; revised 20 April 1977)

Communicated by N. S. Trudinger

\begin{abstract}
The direct method of Lyapunov is utilized to obtain a variety of criteria for the nonexistence of certain types of positive solutions of a delay differential equation of even order. Previous results of Terry (Pacific J. Math. 52 (1974), 269-282) are seen to be corollaries of the more general results of this paper.

Subject classification (Amer. Math. Soc. (MOS) 1970): primary 34 C 10; secondary 34 C 15, 34 K 05, 35 K 15, 34 K 20, 34 K 25.
\end{abstract}

In this paper we consider the general delay differential equation of even order

$$
D^{2 n-i}\left[r(t) D^{i} y(t)\right]+y_{\tau}(t) f\left[t, y_{\tau}(t)\right]=0,
$$

where $0<m \leqslant r(t) \leqslant M<\infty, 0 \leqslant \tau(t) \leqslant T<\infty, y_{\tau}(t)=y[t-\tau(t)]$ and $f(t, u)$ satisfies the following properties:

(F1) $f(t, u)$ is a continuous real valued function on $[0, \infty) \times R$;

(F2) for each fixed $t$ in $[0, \infty), f(t, u)<f(t, v)$ for $0<u<v$;

(F3) for each fixed $t$ in $[0, \infty), f(t, u)>0$ and $f(t,-u)=f(t, u)$ for $u \neq 0$.

We first let

$$
y_{j}(t)= \begin{cases}D^{j} y(t), & j=0, \ldots, i-1, \\ D^{j-i}\left[r(t) D^{i} y(t)\right], & j=i, \ldots, 2 n-1 .\end{cases}
$$

Following Terry (1974), we say that a positive solution $y(t)$ of (1) is of type $B_{j}$ on $\left[T_{0}, \infty\right)$ if for $t \geqslant T_{0}$ the $y_{k}(t)>0(k=0, \ldots, 2 j+1)$ and $(-1)^{k+1} y_{k}(t)>0$ $(k=2 j+2, \ldots, 2 n-1)$. It is of type $B_{j}$ if there is a $T_{0}>0$ such that it is of type $B_{j}$ on $\left[T_{0}, \infty\right)$. As in Terry (1974), it is evident that a positive solution of (1) is necessarily of type $B_{j}$ for some $j=0, \ldots, n-1$. Moreover, the following lemmas may be established. 
LEMMA 1. Let $y(t)$ be a solution of (1) of type $B_{j}$ on $\left[T_{0}, \infty\right)$, where either (i) $i$ is even and $j \leqslant(i-2) / 2$ or (ii) $i$ is odd and $j \leqslant(i-3) / 2$. Then for $t \geqslant T_{1}=T_{0}+T$

$$
\left(t-T_{1}\right) y_{k}(t) \leqslant(2 j+2-k) y_{k-1}(t), \quad k=1, \ldots, 2 j+1 .
$$

LEMMA 2. Let $y(t)$ be a solution of (1) of type $B_{j}$ on $\left[T_{0}, \infty\right)$, where $i$ is odd and $j=(i-1) / 2$. Then for $t \geqslant T_{1}=T_{0}+T$

and

$$
\left(t-T_{1}\right) y_{i}(t) \leqslant M y_{i-1}(t)
$$

$$
\left(t-T_{1}\right) y_{k}(t) \leqslant\left[M m^{-1}+(i-k)\right] y_{k-1}(t), \quad k=1, \ldots, i-1 .
$$

LEMMA 3. Let $y(t)$ be a solution of (1) of type $B_{j}$ on $\left[T_{0}, \infty\right)$, where either (i) $i$ is even and $j \geqslant i / 2$ or (ii) $i$ is odd and $j \geqslant(i+1) / 2$. Then for $t \geqslant T_{1}$

(a) $\left(t-T_{1}\right) y_{k}(t) \leqslant(2 j+2-k) y_{k-1}(t)(k=i+1, \ldots, 2 j+1)$;

(b) $\left(t-T_{1}\right) y_{i}(t) \leqslant M(2 j+2-i) y_{i-1}(t)$;

and

(c) $\left(t-T_{1}\right) y_{k}(t) \leqslant\left[M(2 j+2-i) m^{-1}+(i-k)\right] y_{k-1}(t)(k=1, \ldots, i-1)$.

The proof of each of these three lemmas is elementary using only integration by parts and the definition of a $B_{j}$-solution. The case $i=n$ is considered in Terry (1974), where the three results reduce to Lemmas $2.1,2.2$ and 2.3 respectively.

LEMMA 4. Let $y(t)$ be a solution of (1) of type $B_{j}$ on $\left[T_{0}, \infty\right)$. Then there exist constants $k_{l}>0$ and $t_{l} \geqslant T_{1}$ such that

$$
y_{l}[t-\tau(t)] \geqslant k_{l} y_{l}(t), \quad t \geqslant t, \quad l=0, \ldots, 2 j .
$$

As in Terry (1973), each of the four lemmas may be extended to the case in which $\tau(t)$ satisfies either

(T1) $0 \leqslant \tau(t) \leqslant \mu t, 0 \leqslant \mu<m /(m+M) ;$

or

(T2) $0 \leqslant \tau(t) \leqslant \mu t^{\beta}, 0 \leqslant \mu<\infty$ and $0 \leqslant \beta<1$,

provided the number $T_{1}$ is reinterpreted as $\min \left\{t \geqslant T_{0}: t-\tau(t) \geqslant T_{0}\right.$ for $\left.t \geqslant T_{1}\right\}$.

In this paper we use Lyapunov's second method to obtain criteria for the nonexistence of $B_{j}$-solutions of equation (1). We assume $n \geqslant 2$ and consider the system

$$
\left\{\begin{array}{rlrl}
D y_{k}(t) & =y_{k+1}(t), & & k=0, \ldots, i-2 ; \\
D y_{i-1}(t) & =y_{i}(t) / r(t) ; & & \\
D y_{k}(t) & =y_{k+1}(t), & k=i, \ldots, 2 n-2 ; \\
D y_{2 n-1}(t) & =-y_{\tau}(t) f t, y_{\tau}(t) . & &
\end{array}\right.
$$

By a solution of (2) we mean an ordered $2 n$-tuple $\sigma(t)=\left(y_{0}(t), \ldots, y_{2 n-1}(t)\right)$ which satisfies (2). To simplify the discussion we shall let $R_{a}=[a, \infty), a \geqslant 0 ; R^{*}=(0, \infty)$; $R_{*}=(-\infty, 0) ; R^{1}=R=(-\infty, \infty)$. As in Terry (1974), we shall abbreviate certain 
frequently occurring cartesian products:

$$
\begin{aligned}
R^{p^{*}} & =R^{*} \times \ldots \times R^{*}, \quad p \text { times; } \\
R_{p^{*}} & =R_{*} \times \ldots \times R_{*}, \quad p \text { times; } \\
R_{*}^{*} & =R_{*} \times R^{*} ; R_{*}^{*}=R^{*} \times R_{*} ; \\
R_{a}^{p^{*}} & =R_{a} \times R^{p^{*}} ; \\
\Pi_{j} & =R^{(2 j+1)^{*}} \times\left(R^{*}\right)^{n-1-j} \times R ; \quad \Pi_{j}^{*}=R^{(2 j+1)^{*}} \times\left(R^{*}\right)^{n-1-j} \times R^{*} ; \\
\Pi^{j} & =R_{(2 j+1) *} \times\left(R_{*}^{*}\right)^{n-1-j} \times R ; \quad \Pi^{j}=R_{(2 j+1) *} \times\left(R^{*}\right)^{n-1-j} \times R_{*} .
\end{aligned}
$$

In the following a scalar function $v(t, \sigma(t))$ will be called a Lyapunov function for the system (2) if it is continuous in $(t, o(t))$ in its domain of definition and is locally Lipschitzian in $\sigma(t)$. Following Yoshizawa (1970), we define

$$
\dot{v}_{(1)}(t, \sigma(t))=\limsup _{h \rightarrow 0+} \frac{v(t+h, \sigma(t+h))-v(t, \sigma(t))}{h} \text {. }
$$

THEOREM 1. Suppose that there exist two continuous functions $V(t, \sigma(t))$ and $W(t, \sigma(t))$ which are defined on $R_{T} \times \Pi_{j}$ and $R_{T} \times \Pi^{j}$ respectively for some fixed $T$. Assume further that $V(t, \sigma(t))$ and $W(t, \sigma(t))$ satisfy:

(i) both $V(t, \sigma(t))$ and $W(t, \sigma(t))$ tend to infinity as $t \rightarrow \infty$ uniformly for $\sigma(t) \in \Pi_{j}$ or $\sigma(t) \in \Pi^{j}$ respectively;

(ii) $\dot{V}_{(1)}(t, \sigma(t)) \leqslant 0$ for all sufficiently large $t$, where $\sigma(t)$ is a solution of (2) which for large $t$ lies in the region $\Pi_{j}$; and

(iii) $\dot{W}_{(1)}(t, \sigma(t)) \leqslant 0$ for all sufficiently large $t$, where $\sigma(t)$ is a solution of (2) which for large $t$ lies in the region $\Pi^{j}$.

Then (1) has no solutions of type $B_{j}$. Moreover, (1) has no negative solutions $y(t)$ such that $-y(t)$ is of type $B_{j}$.

Proof. Let $y(t)$ be a solution of (1) of type $B_{j}$. Since $y(t)$ and $y_{1}(t)$ are positive for large $t$, there is a positive $T_{0}$ for which $\sigma(t)$ lies in $\Pi_{j}$ for $t \geqslant T_{0}$. By (ii), for $t$ sufficiently large, for example, for $t \geqslant T_{1} \geqslant T_{0}, V(t, \sigma(t))<V\left(T_{1}, \sigma\left(T_{1}\right)\right)$. On the other hand, condition (i) implies that there is a $T_{2}>T_{1}$ for which $V(t, \sigma(t))>V\left(T_{1}, \sigma\left(T_{1}\right)\right)$ for $t \geqslant T_{2}$, which is a contradiction. By letting $y(t)$ be a negative solution of (1) and considering $W(t, \sigma(t))$, we obtain an analogous contradiction.

Let us assume for the moment that $f(t, u)$ satisfies only (F1).

ThEOREM 2. For $(t, \sigma(t)) \in R_{T} * \times \Pi_{n-1}$ assume that there exists a Lyapunov function $v(t, \sigma(t))$ satisfying:

(i) $y_{2 n-1}(t) v(t, \sigma(t))>0$;

(ii) $\dot{v}_{(1)}(t, \sigma(t)) \leqslant-\lambda(t)$, where $\lambda(t)$ is a continuous function defined on $R_{T}$ such that

$$
\liminf _{t \rightarrow \infty} \int_{T}^{t} \lambda(s) d s \geqslant 0
$$

for $t \geqslant T \geqslant T^{*}$. 
Moreover, suppose that there exists a $T_{1}$ and a function $w(t, \sigma(t))$ which for $(t, \sigma(t))$ in the region $R_{T_{1}} \times R^{(2 n-1)^{*}} \times R_{*}$ is a Lyapunov function satisfying:

(iii) $y_{2 n-1}(t) \leqslant w(t, \sigma(t)) \leqslant b\left(y_{2 n-1}(t)\right)$, where $b(u)$ is a continuous function, $b(0)=0$ and $b(u)<0$ for $u \neq 0$;

and

(iv) $\dot{w}_{(1)}(t, \sigma(t)) \leqslant-\rho(t) w(t, \sigma(t))$, where $\rho(t) \geqslant 0$ is a continuous function such that

$$
\int^{\infty} \exp \left\{-\int_{T}^{t} \rho(s) d s\right\} d t=+\infty \text {. }
$$

If $\sigma(t)$ is a solution of (2) which lies in the region $\Pi_{n-1}$ for sufficiently large values of $t$, then $y_{2 n-1}(t) \geqslant 0$, that is, $\sigma(t) \in \Pi_{n-1}^{*}$.

Proof. Suppose that there is a sequence $\left\{t_{k}\right\}$ for which $t_{k} \rightarrow \infty$ as $k \rightarrow \infty$ and $y_{2 n-1}\left(t_{k}\right)<0$. Assume that $t_{k} \geqslant T^{*}$ and that $t_{k}$ is sufficiently large so that by (4),

$$
\liminf _{t \rightarrow \infty} \int_{l_{k}}^{t} \lambda(s) d s \geqslant 0, \quad t \geqslant t_{k},
$$

and $y_{0}(t), \ldots, y_{2 n-2}(t)$ are positive, where we assume that $n \geqslant 2$. For the case $n=1$, see Yoshizawa (1970). Consider the function $v(t, \sigma(t))$ for $t \geqslant t_{k}$.

$$
\begin{aligned}
v(t, \sigma(t)) & \leqslant v\left(t_{k}, \sigma\left(t_{k}\right)\right)+\int_{t_{k}}^{t} \dot{v}_{(1)}(s, \sigma(s)) d s \\
& \leqslant v\left(t_{k}, \sigma\left(t_{k}\right)\right)-\int_{t_{k}}^{t} \lambda(s) d s .
\end{aligned}
$$

Since $y_{2 n-1}\left(t_{k}\right)<0, v\left(t_{k}, \sigma\left(t_{k}\right)\right)<0$, there is a $T_{1} \geqslant t_{k}$ for which

$$
\int_{t_{k}}^{t} \lambda(s) d s \geqslant v\left(t_{k}, \sigma\left(t_{k}\right)\right) / 2
$$

which implies that for $t \geqslant T_{1}$

$$
v(t, \sigma(t)) \leqslant v\left(t_{k}, \sigma\left(t_{k}\right)\right) / 2<0 .
$$

By (i), $y_{2 n-1}(t)<0$ for $t>T_{1}$. By (iii), there is a $T_{2}>T_{1}$ and a Lyapunov function $w(t, \sigma(t))$ defined on $R_{T_{2}} \times R^{(2 n-1)^{*}} \times R_{*}$. For this $w(t, \sigma(t))$ we have by (iv)

$$
y_{2 n-1}(t) \leqslant w(t, \sigma(t)) \leqslant w\left(T_{2}, \sigma\left(T_{2}\right)\right) \exp \left[-\int_{T_{2}}^{t} \rho(s) d s\right],
$$

where $T_{2}>T_{1}$. By (iii),

$$
y_{2 n-1}(t) \leqslant b\left(y_{2 n-1}\left(T_{2}\right)\right) \exp \left[-\int_{T_{2}}^{t} \rho(s) d s\right] .
$$

Substituting this into the above expression, we get

$$
y_{2 n-1}(u)=\left[y_{2 n-2}(u)\right]^{\prime} \leqslant b\left(y_{2 n-1}\left(T_{2}\right)\right) \exp \left[-\int_{T_{2}}^{u} \rho(s)\right] d s .
$$


Integrating from $T_{2}$ to $t$, we arrive at

$$
y_{2 n-2}(t) \leqslant y_{2 n-2}\left(T_{2}\right)+b\left(y_{2 n-1}\left(T_{2}\right)\right) \int_{T_{2}}^{t} \exp \left[-\int_{T_{2}}^{u} \rho(s) d s\right] d t .
$$

Letting $t \rightarrow \infty$ and using (5), it follows that $y_{2 n-2}(t)<0$ for sufficiently large $t$, which is a contradiction.

By the same argument we can prove the following result.

Theorem 2 . For $(t, \sigma(t)) \in R_{T^{*}} \times \Pi^{n-1}$ assume that there exists a Lyapunov function $v(t, \sigma(t))$ satisfying:

(i) $y_{2 n-1}(t) v(t, \sigma(t))>0$;

and

(ii) $\dot{v}_{(1)}(t, \sigma(t))<-\lambda(t)$, where $\lambda(t)$ is a continuous function defined on $R_{T}$ such that for $t \geqslant T \geqslant T^{*}$

$$
\liminf _{l \rightarrow \infty} \int_{T}^{\imath} \lambda(s) d s \geqslant 0
$$

Moreover, suppose that there exists $a T_{1}$ and a function $w(t, \sigma(t))$ which for $(t, \sigma(t))$ in the region $R_{T_{1}} \times R_{(2 n-1) *} \times R^{*}$ is a Lyapunov function satisfying:

(iii) $y_{2 n-1}(t) \leqslant w(t, \sigma(t)) \leqslant b\left(y_{2 n-1}(t)\right)$, where $b(u)$ is a continuous function, $b(0)=0$ and $b(u)<0$ for $u \neq 0$; and

(iv) $\dot{w}_{(1)}(t, \sigma(t)) \leqslant-\rho(t) w(t, \sigma(t))$, where $\rho(t) \geqslant 0$ is a continuous function for which

$$
\int^{\infty} \exp \left[-\int_{T_{1}}^{t} \rho(s) d s\right] d t=+\infty
$$

If $\sigma(t)$ is a solution of (2) which lies in the region $\Pi^{n-1}$ for sufficiently large values of $t$, then $y_{2 n-1}(t) \leqslant 0$ for large $t$, that is, $\sigma(t) \in \Pi_{*}^{n-1}$.

Remark 1. Since $0<m \leqslant r(t) \leqslant M$, condition (5) is equivalent to

$$
\int^{\infty} \frac{1}{r(t)}\left\{\exp \left[-\int_{x_{1}}^{t} \rho(s) d s\right]\right\} d t=+\infty
$$

To see this we merely note that

$$
\begin{aligned}
M \int^{l} \frac{1}{r(u)} \exp \left[-\int_{T}^{u} \rho(s) d s\right] d u & \geqslant \int^{t} \exp \left[-\int_{T}^{u} \rho(s) d s\right] d u \\
& \geqslant m \int^{t} \frac{1}{r(u)} \exp \left[-\int_{T}^{u} \rho(s) d s\right] d u .
\end{aligned}
$$

In the case $n=1$, we have $v(t, \sigma(t))=v\left(t, y, y^{\prime}\right)$ since $y_{0}=y$ and $y_{1}=r y^{\prime}$. Condition (7) arises naturally in the proof of Theorem 2. For the details see Yoshizawa (1970). 
REMARK 2. Suppose we let $\sigma(t) \equiv 0$ in each of the two theorems. Condition (5) is then trivially valid, and the alternative condition (7) reduces to

$$
\int^{\infty} d t / r(t)=+\infty
$$

Thus, we may replace condition (iv) by $\dot{w}_{(1)}(t, \sigma(t)) \leqslant 0$ and obtain two easy corollaries whose statements are left to the reader.

Remark 3. Let $r(t) \equiv 1$ and $f\left[t, y_{\tau}(t)\right]$ be nonnegative. As already noted, solutions of type $B_{1}$ are solutions of type $A_{1}$ (see Terry, 1974). Theorem 2 asserts that a solution $y(t)$ for which $D^{k} y(t)>0, k=0,1, \ldots, 2 n-2$, must satisfy $D^{2 n-1} y(t)>0$, that is, $y(t)$ must be a solution of type $A_{n-1}$, which is obvious from the lemma of Kiguradze (1962).

Theorem 3. Suppose there are continuous functions $a(t), b(t), \alpha\left(y_{2 n-2}\right)$ and $\beta\left(y_{2 n-2}\right)$ satisfying:

(a) for large $T$,

$$
\underset{t \rightarrow \infty}{\liminf } \int_{T}^{t} a(s) d s \geqslant 0, \quad \liminf _{t \rightarrow \infty} \int_{T}^{t} b(s) d s \geqslant 0 ;
$$

(b) for $u=y_{2 n-2}(t), u \alpha(u)>0$ and $D_{u} \alpha(u) \geqslant 0$, where $y_{k}(t), k=0, \ldots, 2 n-2$, are nonnegative for large $t$;

for $u=y_{2 n-2}(t), u \beta(u)>0$ and $D_{u} \beta(u) \geqslant 0$, where $y_{k}(t), k=0, \ldots, 2 n-2$, are nonpositive for large $t$;

(c) $a(t) \alpha\left[y_{2 n-2}(t)\right] \leqslant f\left[t, y_{\tau}(t)\right] y_{\tau}(t)$ for large $t, y(t) \geqslant 0$;

$b(t) \beta\left[y_{2 n-2}(t)\right] \geqslant f\left[t, y_{\tau}(t)\right] y_{\tau}(t)$ for large $t, y(t) \leqslant 0$.

If $\sigma(t)$ is a solution of (2) which for large $t$ lies in the region $\Pi_{n-1}$, then $y_{2 n-1}(t) \geqslant 0$ for large $t$. If $\sigma(t)$ is a solution of (2) which for large $t$ lies in the region $\Pi^{n-1}$, then $y_{2 n-1}(t) \leqslant 0$ for large $t$.

Proof. Let $\lambda(t)=a(t), \rho(t)=0$ and define $v(t, \sigma(t))$ and $w(t, \sigma(t))$ by

$$
v(t, \sigma(t))=\frac{y_{2 n-1}(t)}{\alpha\left[y_{2 n-2}(t)\right]} ; \quad w(t, \sigma(t))=y_{2 n-1}(t)+\alpha\left[y_{2 n-2}(t)\right] \int_{T}^{t} a(s) d s .
$$

Conditions (i), (ii) and (iii) of Theorem 2 hold. In particular,

(i) $y_{2 n-1}(t) v(t, \sigma(t))=\frac{y_{2 n-1}^{2}(t)}{\alpha\left[y_{2 n-2}(t)\right]}>0 \quad$ since $y_{2 n-2}(t)>0$;

(ii) $\dot{v}_{(1)}(t, \sigma(t))=\left\{\alpha D y_{2 n-1}(t)-y_{2 n-1}^{2}(t) \alpha^{\prime}\left(y_{2 n-2}(t)\right)\right\} / \alpha^{2}\left(y_{2 n-2}(t)\right)$

$$
\begin{array}{ll}
<\frac{D y_{2 n-1}(t)}{\alpha\left[y_{2 n-2}(t)\right]} & \text { (by condition (b)) } \\
=\frac{-f\left[t, y_{\tau}(t)\right] y_{\tau}(t)}{\alpha\left[y_{2 n-2}(t)\right]} & (\text { from (1)) } \\
\leqslant-a(t)=-\lambda(t) & \text { (by condition (a)). }
\end{array}
$$


Moreover,

$$
\liminf _{t \rightarrow \infty} \int_{T}^{t} \lambda(s) d s=\liminf _{t \rightarrow \infty} \int_{T}^{t} a(s) d s \geqslant 0
$$

for large $t$ by condition (a).

(iii) $y_{2 n-1}(t) \leqslant w(t, \sigma(t)) \leqslant y_{2 n-1}(t)+\alpha\left[y_{2 n-2}(t)\right] \int_{T}^{t} a(s) d s$, since $y_{2 n-2}(t) \geqslant 0$. Also,

$$
\begin{aligned}
\alpha\left[y_{2 n-2}(t)\right] \int_{T}^{t} a(s) d s & \leqslant \int_{T}^{t} \alpha\left[y_{2 n-2}(s)\right] d s \\
& \leqslant \int_{T}^{t} f\left[s, y_{\tau}(s)\right] y_{\tau}(s) d s \\
& =y_{2 n-1}(T)-y_{2 n-1}(t) .
\end{aligned}
$$

For, if we assume as in (iii) of Theorem 2 that $\sigma(t) \in R^{(2 n-1)^{*}} \times R_{*}, y_{2 n-2}(t)$ is a positive decreasing function of $t$. Thus, for $s<t$,

$$
y_{2 n-2}(t)<y_{2 n-2}(s) \text { and } \alpha\left[y_{2 n-2}(t)\right]<\alpha\left[y_{2 n-2}(s)\right]
$$

since $D_{u} \alpha(u)>0$. It follows that $w(t, \sigma(t)) \leqslant y_{2 n-1}(T)<0$. Thus, we may take $b(u)$ to be the continuous function for which $b(0)=0, b(u)=y_{2 n-1}(T)$ for $u \notin(-\varepsilon, \varepsilon)$ and $b(u)$ is defined linearly on $(-\varepsilon, \varepsilon)$.

Moreover, to prove the second assertion of the theorem, suppose we let $v(t, \sigma(t))$ and $w(t, \sigma(t))$ be defined by

$$
v(t, \sigma(t))=\frac{y_{2 n-1}(t)}{\beta\left[y_{2 n-2}(t)\right]} ; \quad w(t, \sigma(t))=-y_{2 n-1}(t)-\beta\left[y_{2 n-1}(t)\right] \int_{T}^{t} b(s) d s .
$$

Routine computations, similar to those just performed, show that $v(t, \sigma(t))$ and $w(t, \sigma(t))$ satisfy the four conditions of Theorem $2^{\prime}$.

THEOREM 4. Suppose that, in addition to the hypotheses of Theorem 3,

$$
\int^{\infty} a(s) d s=\int^{\infty} b(s) d s=+\infty .
$$

Then (1) has no solutions of type $B_{n-1}$.

Proof. Suppose we define

$$
\begin{gathered}
V(t, \sigma(t))=\left\{\begin{array}{rr}
\frac{y_{2 n-1}(t)}{\alpha\left[y_{2 n-2}(t)\right]}+\int_{0}^{t} a(s) d s, & y \geqslant 0, \\
\int_{0}^{t} a(s) d s, & y<0 ;
\end{array}\right. \\
W(t, \sigma(t))=\left\{\begin{array}{rr}
\frac{y_{2 n-1}(t)}{\beta\left[y_{2 n-2}(t)\right]}+\int_{0}^{t} b(s) d s, & y<0, \\
\int^{t} b(s) d s, & y \geqslant 0 .
\end{array}\right.
\end{gathered}
$$


Assume that $y(t)$ is a solution of (1) of type $B_{n-1}$. Then for large $t, y_{k}(t) \geqslant 0$ for $k=0, \ldots, 2 n-1$. It follows that

$$
V(t, \sigma(t)) \geqslant \int_{0}^{t} a(s) d s \text { and } W(t, \sigma(t)) \geqslant \int_{0}^{t} b(s) d s .
$$

Because of the additional requirement in the hypothesis of this theorem, both $V(t, \sigma(t))$ and $W(t, \sigma(t))$ tend to infinity as $t \rightarrow \infty$ uniformly. Next, referring to (ii) of the proof of Theorem 3

$$
\begin{aligned}
& \dot{V}_{(1)}(t, \sigma(t))=D \frac{y_{2 n-1}(t)}{\alpha\left[y_{2 n-2}(t)\right]}+a(t) \leqslant 0 ; \\
& \dot{W}_{(1)}(t, \sigma(t))=D \frac{y_{2 n-1}(t)}{\beta\left[y_{2 n-2}(t)\right]}+b(t) \leqslant 0 .
\end{aligned}
$$

Hence, $V(t, \sigma(t))$ and $W(t, \sigma(t))$ satisfy the three conditions of Theorem 1 and the proof is complete. As in Theorem 1, we may also conclude that there are no negative solutions $y(t)$ of (1) such that $-y(t)$ is of type $B_{n-1}$.

We observe that Theorem 4 is only one of a sequence of similar results. Let us now consider the more general formulation.

THEOREM 5. Suppose that there are continuous functions $a(t), b(t), \alpha(u)$ and $\beta(u)$ satisfying:

(a)

$$
\int^{\infty} a(s) d s=\int^{\infty} b(s) d s=+\infty ;
$$

(b) $u \alpha(u)>0, D_{u} \alpha(u) \geqslant 0$, where $u$ and $u^{\prime}$ are nonnegative for large $t$; $u \beta(u)>0, D_{u} \beta(u) \geqslant 0$, where $u$ and $u^{\prime}$ are nonpositive for large $t$;

(c) $a(t) \alpha\left[y_{2 j}(t)\right] \leqslant f\left[t, y_{\tau}(t)\right] y_{\tau}(t)$ and $b(t) \beta\left[y_{2 j}(t)\right] \leqslant f\left[t, y_{\tau}(t)\right] y_{\tau}(t)$. Then (1) has no solutions of type $B_{r}(r=j, \ldots, n-1)$.

Proof. Let

$$
\begin{gathered}
V(t, \sigma(t))=\left\{\begin{array}{c}
\frac{y_{2 n-1}(t)}{\alpha\left[y_{2 j}(t)\right]}+\int_{0}^{t} a(s) d s, \quad y<0 \\
\int_{0}^{t} a(s) d s, y=0
\end{array}\right. \\
W(t, \sigma(t))=\left\{\begin{aligned}
\frac{y_{2 n-1}(t)}{\beta\left[y_{2 j}(t)\right]}+\int_{0}^{t} b(s) d s, y>0 \\
\int_{0}^{t} b(s) d s, \quad y \leqslant 0 .
\end{aligned}\right.
\end{gathered}
$$

As in the proof of Theorem $4, V(t, \sigma(t))$ and $W(t, \sigma(t))$ will satisfy the three conditions of Theorem 1. The details are omitted. Moreover, there are no negative solutions $y(t)$ of (1) such that $-y(t)$ is of type $B_{r}(r=j, \ldots, n-1)$. 
COROLLARY 1. Let $p(t)>0$. If $\int^{\infty} t^{2 j} p(t) d t=+\infty$, then there are no solutions of (8)

$$
D^{n}\left[r(t) D^{n} y(t)\right]+p(t) y_{\tau}(t)=0
$$

of type $B_{r}(r=j, \ldots, n-1)$.

Proof. Let $y(t)$ be a solution of (8) of type $B_{r}(r=j, \ldots, n-1)$. Then

$$
f\left[t, y_{\tau}(t)\right] y_{\tau}(t)=p(t) y_{\tau}(t) \geqslant \mu t^{2 j} p(t) y_{2 j}(t) .
$$

We let $\alpha(u)=\beta(u)=\mu u$ and $\lambda(t)=a(t)=b(t)=t^{2 j} p(t)$. With the choices of $V(t, \sigma(t))$ and $W(t, \sigma(t))$ prescribed by Theorem 4 , it follows that (8) has no solutions of type $B_{r}$.

\section{REFERENCES}

I. T. Kiguradze (1962), "Oscillation properties of solutions of certain ordinary differential equations”, Dokl. Akad. Nauk SSSR 144, 33-36; translated in Soviet Math. Dokl. 3 (1962), 649-652.

R. D. Terry (1973), “Oscillatory properties of a fourth-order delay differential equation, 2", Funkcial. Ekvac. 16, 213-224.

R. D. Terry (1974), "Oscillatory properties of a delay differential equation of even order", Pacific J. Math. 52, 269-282.

R. D. Terry (1975), "Some oscillation criteria for delay differential equations of even order", SIAM J. Appl. Math. 28, 319-334.

T. Yoshizawa (1970), "Oscillation property of solutions of second order differential equations", Tohoku Math. J. 22, 619-634.

California Polytechnic State University

San Luis Obispo, California 93407

U.S.A. 